\title{
Multi-criteria Credibilistic Portfolio Selection Model with Various Risk Comparisons Using Trapezoidal Fuzzy Variable
}

\author{
Jagdish Kumar Pahade*, Manoj Jha \\ Department of Mathematics, Maulana Azad National Institute of Technology, Bhopal, India \\ Email address: \\ jkp.173104001@manit.ac.in(J. K. Pahade),m_jha28@rediffmail.com (M. Jha) \\ ${ }^{*}$ Corresponding author
}

To cite this article:

Jagdish Kumar Pahade, Manoj Jha. Multi-criteria Credibilistic Portfolio Selection Model with Various Risk Comparisons Using Trapezoidal Fuzzy Variable. Applied and Computational Mathematics. Vol. 0, No. 1, 2021, pp. 1-9. doi: 10.11648/j.acm.20211001.11

Received: January 12, 2021; Accepted: January 19, 2021; Published: February 10, 2021

\begin{abstract}
Dealing with problems on portfolio selection models fuzzy set theory is effectively interpolating investor's attitude. The credibility theory (Branch of fuzzy set theory) is broadly utilized to describe uncertainty of the financial markets. We regard the return rate of each risky stock as a trapezoidal fuzzy number. Variance and semi-variance of fuzzy return on stocks are widely accepted as risk measures in portfolio selection models. This paper obtains credibilistic semi-variance of trapezoidal fuzzy variable and applied this concept to quantify the risk in stock fuzzy portfolio selection. A multi-criteria credibilistic mean-semivariance-skewness model is proposed with numerical illustration taking historical data set from the premier market for financial assets. Three objectives are taken into account namely, expected portfolio return, risk on expected portfolio return and portfolio skewness to construct multi-objective programming problem, along with cardinality constraint, complete capital utilization, floor and ceiling constraint, no short selling constraints. To solve the proposed multi-objective optimization problem, optimal goal programming approach is suggested. Finally, a case study is conducted to highlight the effectiveness of the proposed models through the real-world data from the Bombay Stock Exchange (BSE), an Indian premier market for financial stocks. Furthermore, results comparison of semi-variance as risk measure with other existing risk measures is performed.
\end{abstract}

Keywords: Trapezoidal Fuzzy Variables, Credibilistic Semi-variance, Fuzzy Portfolio Selection, Optimal Goal Programming

\section{Introduction}

Modern theory of portfolio optimization is an improvement of milestone research of Markowitz [1] namely "mean-variance model". The variance as a tool of risk measure comes to be very popular in portfolio theory. However, variance is criticized also because variance considers equal weight to both low and high returns. Markowitz [2] rectified this limitation of variance by introducing a new risk measure namely "semi-variance" in the portfolio optimization theory. Some scholars gathered merits of semi-variance measuring risk in portfolio optimization [3-5]. These works were proposed based on the assumption that returns on risky stocks are random variables and correctly reflected by available real historical data. In general, the stock market is so complex and suffers from the lack of larger data because of the occurrence of new stocks. There may be some cases such that the stock returns are surrounded with ambiguity and vagueness in the stock market see [6, 7]. To handle such situations modern researcher suggests describing stock returns by fuzzy sets or fuzzy numbers see $[8,9]$ is applied possibility measure to describe parameters. Zhang [16] presented multi-period model for fuzzy portfolio selection taking risk as possibilistic semi-variance of trapezoidal fuzzy variable with various constraints. Yong and Zhang [15] proposed investor's different behavior portfolio optimization model based on possibilistic semi-variance of LR fuzzy number. Liu et al. [17] presented fuzzy portfolio performance evaluation based multi-period model using possibilistic semi-variance with 
some realistic constraints. Although the theory of possibility has been widely adopted, it does not follow the theory of protection of truth and is incompatible with the principle of abstinent central and the theory of contraposition. The major argument is that possibility measure does not follow selfduality property. Although a self-dual property is thoroughly expected both in practice and theory. Liu and Liu [10] introduced new credibility measure to overcome the limitations of possibility measure which is being broadly applied in modern theory of fuzzy portfolio optimization. Huang [11] firstly defined semi-variance of fuzzy variables based on credibility theory and applied this concept to manage the risk in fuzzy portfolio optimization. Qin et al. [12] proposed cross-entropy minimization model for fuzzy portfolio optimization by taking risk credibilistic variance and semi-variance. Qin et al. [13] defined uncertain and random semi-variance for asymmetric random fuzzy return to measure downside risk and formulate fuzzy portfolio optimization problem. Jalota et al. [14] obtained credibilistic semi-variance of L-R power fuzzy variable and employed this semi-variance to compute downside risk in fuzzy portfolio optimization with multi-criteria. Zhang and Peng [18] presented multi-period model using credibilistic semivariance of triangular fuzzy variable with transaction cost and various constrains.

Above cited research works reviled the utility of semivariance as risk measure in portfolio theory especially in fuzzy portfolio selection problems. Presented work in this paper is a motivation of sapidity of researchers in modern portfolio theory. The key differences of presented research work from existing work are disquisition of credibilistic semi-variance of trapezoidal fuzzy variable when credibilistic mean returns lie within the cores and typical extension of mean-semivariance model to meansemivariance-skewness model for credibilistic portfolio optimization. Modern research works have been presented portfolio selection models assuming that returns on risky stocks are trapezoidal fuzzy variable under credibilistic framework such as Mehlawat, M. K. [19] presented meanentropy model and suggested multi-choice goal programming approach to select portfolios, Vercher and Bermudez [20] proposed rank-index model based on expected mean and loss function, Liu et al. [21] introduced $\mathrm{CVaR}$ as a new risk and presented mean-conditional valueat-risk model, Gupta P. et al. [22] proposed data envelopment analysis model for fuzzy portfolio selection, Mehlawat, M. K. et al. [23] proposed multi-objective portfolio performance evaluation model credibilistic framework etcetera. After defining skewness of fuzzy variable and considering in fuzzy portfolio selection by Li et al. [24], lots of research works have presented and argued about the role of skewness on portfolio selection under fuzzy environment see [25-30].

Credibilistic semi-variance is not only risk measure in fuzzy portfolio optimization theory but there are some other risk measures are also notified by researchers such as credibilistic variance, credibilistic absolute deviation, credibilistic entropy, credibilistic Value-at-risk etc see [3134]. Although, these risk measures have organized importance in fuzzy portfolio selection not enough compatible to compare with semi-variance, but there are some more compatible risk measures to compare with semivariance such as credibilistic semi-absolute deviation, credibilistic semi-entropy and credibilistic conditional valueat-risk see [35, 21, 27]. Therefore we will compare these three risk measures with presented risk measure for fuzzy portfolio selection problem.

Rest of the paper is organized in the following sections: Section 2 reviewed some necessary basic definitions and essential properties for credibilistic measure of fuzzy variables. In section 3 , the credibilistic semi-variance of the trapezoidal fuzzy variable is obtained. In section 4, we presented mean-semivariance-skewness model with solution methodology of the multi-objective programming problem for fuzzy portfolio selection. In section 5, a numerical example is presented to illustrate the performance of the proposed model and results comparison with the existing models. In section 6 , the paper has been concluded by putting some comments. Acknowledgments and references are presented in the last of the paper.

\section{Preliminaries}

In this section, we recall the credibilistic notion and some basic concepts relevant to continuous fuzzy variable. In order to measure fuzzy sets, Zadeh [36] introduced a possibility measure. Fuzzy portfolio selection based on possibility measure become very popular, but criticized due to the absence of duality property in possibility measure, Liu and Liu [10] introduced credibility measure for fuzzy variables with duality property which is necessary in theory and practical. Some necessary basic definitions and essential properties for credibilistic measure of fuzzy variables are as follows:

Definition 2.1 ( $\mathrm{Li}$ and Liu [37]): For the power set $\mathbb{P}$ of a non-empty set??, each member $A \in \mathbb{P}$ is called an event and for any two members $\mathrm{A}, \mathrm{B} \in \mathbb{P}$ the set function $\operatorname{Cr}\{A\}$, such that $0 \leq \operatorname{Cr}\{A\} \leq 1$, is called the credibility measure if following axioms are hold

Normality axiom: $\operatorname{Cr}\{\Theta\}=1$.

Monotonicity axiom: $\operatorname{Cr}\{A\} \leq \operatorname{Cr}\{B\}$, whenever $A \subset B$.

Self-duality axiom: $\operatorname{Cr}\{A\}+\operatorname{Cr}\left\{A^{c}\right\}=1$, for any event $A$.

Maximality axiom: $r\left\{\cup_{i} A_{i}\right\}=\sup _{i} \operatorname{Cr}\left\{A_{i}\right\}$, for any sequence of event $\left\{A_{i}\right\}$ ith $\sup _{i} \operatorname{Cr}\left\{A_{i}\right\}<0.5$.

Definition 2.2 (Liu [38]): The membership function $\mu(t)$ of a fuzzy variable $\eta$ can be derived from the credibility measure as follows:

$$
\mu(t)=(2 \operatorname{Cr}\{\eta=t\}) \wedge 1, t \in R .
$$

Definition 2.3 (Liu [38]): For a fuzzy variable $\eta$ with membership function $\mu(t)$, we have

$$
\operatorname{Cr}\{\eta \in A\}=\frac{1}{2}\left(\sup _{t \in A} \mu(t)+1-\sup _{t \in A^{c}} \mu(t)\right)
$$


Definition 2.4 (Liu and Liu [10]): The expected value of a fuzzy variable $\eta$ can be defined by

$$
E(\eta)=\int_{0}^{+\infty} \operatorname{Cr}\{\eta \geq r\} d r-\int_{-\infty}^{0} \operatorname{Cr}\{\eta \leq r\} d r
$$

Definition 2.5 (Liu and Liu [10]): The variance of a fuzzy variable $\eta$ with finite expected value $E(\eta)$ can be defined by

$$
V(\eta)=E\left[(\eta-E(\eta))^{2}\right]
$$

Definition 2.6 (Liu [39]): The credibility distribution $\Gamma: R \rightarrow[0,1]$ of a fuzzy variable $\eta$ can be defined by

$$
\Gamma(r)=\operatorname{Cr}\{\theta \in \Theta \mid \eta(\theta) \leq r\}
$$

Definition 2.7 ( $\mathrm{Li}$ et al. [24]): Let $\eta$ be a fuzzy variable with finite expected value $E[\eta]$. Then its skewness can be defined by

$$
S_{k}[\eta]=E\left[(\eta-E[\eta])^{3}\right]
$$

Example 2.1: Let $\operatorname{Trap}(\eta)=\left(t_{a}, t_{b}, t_{c}, t_{d}\right)$ be a trapezoidal fuzzy variable, then its credibility distribution can be given by

$$
\Gamma(r)=\left\{\begin{array}{c}
0, \text { if } r<t_{a} \\
\frac{r-t_{a}}{2\left(t_{b}-t_{a}\right)}, \text { if } t_{a} \leq r<t_{b}, \\
\frac{1}{2}, \text { if } t_{b} \leq r<t_{c}, \\
\frac{t_{d}-2 t_{c}+r}{2\left(t_{d}-t_{c}\right)}, \text { if } t_{c} \leq r<t_{d}, \\
1, \text { if } r \geq t_{d} .
\end{array}\right.
$$

Example 2.2: The credibilistic expected value of trapezoidal fuzzy variable $\operatorname{Trap}(\eta)=\left(t_{a}, t_{b}, t_{c}, t_{d}\right)$ can be given by

$$
E[\eta]=\frac{t_{a_{i}}+t_{b_{i}}+t_{c_{i}}+t_{d_{i}}}{4}
$$

Theorem 2.1 (Liu and Liu [40]): Let $\eta$ be a fuzzy variable with finite expected value $E(\eta)$. Then for any two real numbers $r_{1}$ and $r_{2}$ we have

$$
\begin{aligned}
& E\left(r_{1} \eta+r_{2}\right)=r_{1} E(\eta)+r_{2} \\
& \left\{\begin{array}{c}
\frac{1}{2}, \text { if } 0 \leq r \leq \frac{t_{a}-3 t_{b}+t_{c}+t_{d}}{4}, \\
\frac{-3 t_{a}+t_{b}+t_{c}+t_{d}-4 r}{8\left(t_{b}-t_{a}\right)}, \text { if } \frac{t_{a}-3 t_{b}+t_{c}+t_{d}}{4} \leq r \leq \frac{-3 t_{a}+t_{b}+t_{c}+t_{d}}{4}, \\
0, \text { if } r \geq \frac{-3 t_{a}+t_{b}+t_{c}+t_{d}}{4} .
\end{array}\right.
\end{aligned}
$$

Using this credibility in mentioned formula of variance, we get the variance of trapezoidal fuzzy variable as follows:

$$
\begin{gathered}
V_{s}(\eta)=\int_{0}^{\frac{t_{a}-3 t_{b}+t_{c}+t_{d}}{4}} \frac{1}{2} d r+\int_{\frac{t_{a}-3 t_{b}+t_{c}+t_{d}}{4}}^{\frac{-3 t_{a}+t_{b}+t_{c}+t_{d}}{4}} \frac{-3 t_{a}+t_{b}+t_{c}+t_{d}-4 r}{8\left(t_{b}-t_{a}\right)} d r \\
=\frac{1}{96}\left[7\left(t_{b}-t_{a}\right)^{2}+3\left(t_{d}-t_{c}\right)^{2}+6\left(t_{b}-t_{a}\right)\left(t_{d}-t_{c}\right)+12\left(t_{d}-t_{a}\right)\left(t_{c}-t_{b}\right)\right]
\end{gathered}
$$

Theorem 2.2 (Liu and Liu [40]): Let $\eta_{1}$ and $\eta_{2}$ be two fuzzy variables with finite expected values $E\left(\eta_{1}\right)$ and $E\left(\eta_{2}\right)$ respectively. Then for any two real numbers $r_{1}$ and $r_{2}$ we have

\section{Credibilistic Semi-variance of the Trapezoidal Fuzzy Variable}

In order to obtain credibilistic semi-variance for trapezoidal fuzzy variable, we define semi-variance for fuzzy variable and its mathematical representation in terms of credibility measure. Let $\eta$ be a fuzzy variable with finite expected value $E(\eta)$, then semi-variance of $\eta$ can be defined as

where

$$
(\eta-E[\eta])^{-}=\left\{\begin{array}{c}
\eta-E[\eta], \text { if } \eta \leq E[\eta] \\
0, \text { if } \eta \geq E[\eta] .
\end{array}\right.
$$

It clear from the definition of semi-variance that $V_{S}(\eta)$ is always no-negative for fuzzy variable $\eta$. Using the definition 2.4 following mathematical representation of $V_{s}(n)$ is formulated.

$$
\begin{aligned}
V_{s}(\eta) & =\int_{0}^{\infty} \operatorname{Cr}\left\{\left((\eta-E[\eta])^{-}\right)^{2} \geq r\right\} d r \\
& =\int_{0}^{\infty} \operatorname{Cr}\left\{\left|(\eta-E[\eta])^{-}\right| \geq \sqrt{r}\right\} d r \\
& =2 \int_{0}^{\infty} r \operatorname{Cr}\left\{\left|(\eta-E[\eta])^{-}\right| \geq r\right\} d r \\
& =2 \int_{0}^{\infty} r \operatorname{Cr}\{\eta \leq E[\eta]-r\} d r
\end{aligned}
$$

This shows that credibility measure $\operatorname{Cr}\{\eta \leq E[\eta]-r\}$ for non-negative $r$ is required to obtain $V_{s}(\eta)$, which can be obtained from the definition 2.1 for trapezoidal fuzzy variable $\operatorname{Trap}(\mathrm{n})=\left(t_{a}, t_{b}, t_{c}, t_{d}\right)$ as follows: 
Theorem 3.1 - Assume that $\operatorname{Trap}(\eta)=\left(t_{a}, t_{b}, t_{c}, t_{d}\right)$ be a trapezoidal fuzzy variable with finite expected value $E[\eta]$ such that $b \leq E[\eta] \leq c$, then

i. Credibilistic skewness $S_{k}[\eta]$ of $\operatorname{Trap}(\eta)$ is given as follows:

$$
S_{k}[\eta]=\frac{1}{32}\left[\left(t_{d}-t_{c}\right)^{2}-\left(t_{b}-t_{a}\right)^{2}\right]\left[t_{d}+t_{c}-t_{b}-t_{a}\right]
$$

ii. Credibilistic semi-absolute deviation $S A D[\eta]$ of $\operatorname{Trap}(\mathrm{\eta})$ is given as follows:

$$
\begin{array}{r}
S A D[\mathrm{\eta}]=\frac{t_{d}+t_{c}-t_{b}-t_{a}}{8} \quad\left(x_{1}, x_{2}, \ldots, x_{n}\right) \text { is also a trapezoidal } \mathrm{u} z \\
\eta=\eta_{1} x_{1}+\eta_{2} x_{2}+\cdots+\eta_{n} x_{n}=\left(\sum_{i=1}^{n} t_{a_{i}} x_{i}, \sum_{i=1}^{n} t_{b_{i}} x_{i}, \sum_{i=1}^{n} t_{c_{i}} x_{i}, \sum_{i=1}^{n} t_{d_{i}} x_{i}\right)
\end{array}
$$

such that its credibilistic expected value lies within the core. Experts' knowledge allows us to incorporate the expected value of the trapezoidal fuzzy variables between the cores because of the maximum membership grade in it. To obtain a multi-objective programming problem the following objective functions and constraints are constructed.

\section{Credibilistic Mean- semivariance-skewness Model}

In order to construct an investment portfolio under multiobjective fuzzy portfolio selection, let us consider $n$ risky stocks in the financial stock market. Assume that future return rates of stocks are independent trapezoidal fuzzy variables $\eta_{i}=\left(t_{a_{i}}, t_{b_{i}}, t_{c_{i}}, t_{d_{i}}\right), i=1,2, \ldots, n$, with real continuous membership functions. Suppose that $x_{i}, i=$ $1,2, \ldots, n$, be proportions of investing budget for various stocks. Note that the future return of the portfolio $x=$ $\left(x_{1}, x_{2}, \ldots, x_{n}\right)$ is also a trapezoidal fuzzy variable

\subsection{Objective Functions}

$$
E\left(\eta_{1} x_{1}+\eta_{2} x_{2}+\cdots+\eta_{n} x_{n}\right)=\frac{\sum_{i=1}^{n} t_{a_{i}} x_{i}+\sum_{i=1}^{n} t_{b_{i}} x_{i}+\sum_{i=1}^{n} t_{c_{i}} x_{i}+\sum_{i=1}^{n} t_{d_{i}} x_{i}}{4}=\sum_{i=1}^{n}\left(\frac{t_{a_{i}}+t_{b_{i}}+t_{c_{i}}+t_{d_{i}}}{4}\right) x_{i}
$$

\section{Risk on portfolio return}

\section{Expected portfolio return}

Since portfolio return is a trapezoidal fuzzy variable, using the credibilistic expected value of trapezoidal fuzzy variable as shown in example 2.2 the expected portfolio return is obtained as:

Using credibilistic semi-variance of trapezoidal fuzzy variable shown as in section 3 , the risk on portfolio return

$$
\frac{1}{96}\left\{\begin{aligned}
7\left[\sum_{i=1}^{n}\left(t_{b_{i}}-t_{a_{i}}\right) x_{i}\right]^{2} & +3\left[\sum_{i=1}^{n}\left(t_{d_{i}}-t_{c_{i}}\right) x_{i}\right]^{2}+6\left[\sum_{i=1}^{n}\left(t_{b_{i}}-t_{a_{i}}\right) x_{i}\right] \cdot\left[\sum_{i=1}^{n}\left(t_{b_{i}}-t_{a_{i}}\right) x_{i}\right] \\
& +12\left[\sum_{i=1}^{n}\left(t_{b_{i}}-t_{a_{i}}\right) x_{i}\right] \cdot\left[\sum_{i=1}^{n}\left(t_{b_{i}}-t_{a_{i}}\right) x_{i}\right]
\end{aligned}\right\}
$$

The skewness of the portfolio

Using credibilistic skewness of trapezoidal fuzzy variable according to the theorem 5.1 the skewness of portfolio $S_{k}\left(\eta_{1} x_{1}+\right.$ $\left.\eta_{2} x_{2}+\cdots+\eta_{n} x_{n}\right)$ is obtained as:

$$
\frac{1}{32}\left\{\left[\sum_{i=1}^{n}\left(t_{d_{i}}-t_{c_{i}}\right) x_{i}\right]^{2}-\left[\sum_{i=1}^{n}\left(t_{b_{i}}-t_{a_{i}}\right) x_{i}\right]^{2}\right\}\left\{\sum_{i=1}^{n}\left(t_{d_{i}}+t_{c_{i}}-t_{b_{i}}-t_{a_{i}}\right) x_{i}\right\}
$$

\subsection{Constrains}

Constraint of the complete capital budget on the stocks:

$$
\sum_{i=1}^{n} x_{i}=1
$$

(Floor and ceiling constraint) -Maximum fraction of the capital budget that can be invested in a separate stock:

$$
x_{i} \leq u_{i} y_{i}, \forall i=1,2, \ldots, n,
$$

Infinitesimal fraction of the capital budget that can be invested in a separate stock:

$$
x_{i} \geq l_{i} y_{i}, \forall i=1,2, \ldots, n .
$$

Selection or rejection of stocks in the portfolio:

$$
y_{i}=\left\{\begin{array}{c}
1, \text { if } i \text { th stock is included in the portfolio, } \\
0, \text { otherwise }
\end{array}\right.
$$

The minimum number (cardinality constraint) of stocks 
held in the portfolio:

$$
\sum_{i=1}^{n} y_{i} \geq 4,
$$

No short-selling of stocks:

$$
0 \leq x_{i} \leq 1, \forall i=1,2, \ldots, n .
$$

\subsection{The Multi-objective Optimization Problem}

Suppose that an investor wants to maximize expected portfolio return, minimize the portfolio risk and maximize the portfolio skewness simultaneously under some boundary conditions. Using the above objective functions and constraints, we proposed the following multi-objective MSVS model for fuzzy portfolio selection:

$$
\left\{\begin{array}{c}
\max E\left(\eta_{1} x_{1}+\eta_{2} x_{2}+\cdots+\eta_{n} x_{n}\right) \\
\min V_{S}\left(\eta_{1} x_{1}+\eta_{2} x_{2}+\cdots+\eta_{n} x_{n}\right) \\
\max S_{k}\left(\eta_{1} x_{1}+\eta_{2} x_{2}+\cdots+\eta_{n} x_{n}\right) \\
\text { s.t. } x_{1}+x_{2}+\cdots+x_{n}=1 \\
y_{1}+y_{2}+\cdots+y_{n} \geq 4 \\
l_{i} y_{i} \leq x_{i} \leq u_{i} y_{i}, i=1,2, \ldots, n, \\
0 \leq x_{i} \leq 1, i=1,2, \ldots, n, \\
y_{i} \in\{0,1\}
\end{array}\right.
$$

\subsection{Solution Methodology}

To solve the multi-objective programming problems, various approaches have been proposed in the literature like genetic algorithm, particle swarm optimization and goal programming. In this paper, optimal goal programming has been applied to solve multi-objective programming problem. All the three objective functions corresponding to expected portfolio return, risk on portfolio return and portfolio skewness can be solved separately with constraints to assign the optimal goal to the objective function as follows:

I.

$$
\left\{\begin{array}{c}
\max E\left(\eta_{1} x_{1}+\eta_{2} x_{2}+\cdots+\eta_{n} x_{n}\right)=g_{1} \\
\text { s.t. } x_{1}+x_{2}+\cdots+x_{n}=1 \\
y_{1}+y_{2}+\cdots+y_{n} \geq 4 \\
l_{i} y_{i} \leq x_{i} \leq u_{i} y_{i}, i=1,2, \ldots, n \\
0 \leq x_{i} \leq 1, i=1,2, \ldots, n \\
y_{i} \in\{0,1\}
\end{array}\right.
$$

II. $\left\{\begin{array}{c}\min V_{S}\left(n_{1} x_{1}+n_{2} x_{2}+\cdots+n_{n} x_{n}\right)=g_{2} \\ \text { s.t. } x_{1}+x_{2}+\cdots+x_{n}=1 \\ y_{1}+y_{2}+\cdots+y_{n} \geq 4 \\ l_{i} y_{i} \leq x_{i} \leq u_{i} y_{i}, i=1,2, \ldots, n \\ 0 \leq x_{i} \leq 1, i=1,2, \ldots, n \\ y_{i} \in\{0,1\}\end{array}\right.$

$$
\text { III. }\left\{\begin{array}{c}
\max S_{k}\left(\eta_{1} x_{1}+\eta_{2} x_{2}+\cdots+\eta_{n} x_{n}\right)=g_{3} \\
\text { s.t. } x_{1}+x_{2}+\cdots+x_{n}=1 \\
y_{1}+y_{2}+\cdots+y_{n} \geq 4 \\
l_{i} y_{i} \leq x_{i} \leq u_{i} y_{i}, i=1,2, \ldots, n \\
0 \leq x_{i} \leq 1, i=1,2, \ldots, n \\
y_{i} \in\{0,1\}
\end{array}\right.
$$

After solving these three problems and assigning optimal goals $g_{1}, g_{2}$, and $g_{3}$ to the objective functions the multi- objective programming problem can be reformulated to a single objective programming problem as follows:

$$
\left\{\begin{array}{c}
\min d_{1}+d_{2}+d_{3} \\
\text { s.t } E\left(\eta_{1} x_{1}+\eta_{2} x_{2}+\cdots+\eta_{n} x_{n}\right)+d_{1}=g_{1} \\
V_{S}\left(\eta_{1} x_{1}+\eta_{2} x_{2}+\cdots+\eta_{n} x_{n}\right)-d_{2}=g_{2} \\
S_{k}\left(\eta_{1} x_{1}+\eta_{2} x_{2}+\cdots+\eta_{n} x_{n}\right)+d_{3}=g_{3} \\
x_{1}+x_{2}+\cdots+x_{n}=1 \\
y_{1}+y_{2}+\cdots+y_{n} \geq 4 \\
l_{i} y_{i} \leq x_{i} \leq u_{i} y_{i}, i=1,2, \ldots, n, \\
0 \leq x_{i} \leq 1, i=1,2, \ldots, n, \\
y_{i} \in\{0,1\}
\end{array}\right.
$$

This single objective optimization problem with all constraints is main programming problem to get proposed investment strategies, which can be solved easily.

\section{Numerical Experimental Analysis with Discussion}

In this section, we present and discuss the outcomes of an experimental screening for which we trust on real data set of weekly closing prices of stocks listed in Indian premier market for financial stocks.

\subsection{Input Data Description}

To investigate the performance of the proposed model for fuzzy portfolio selection, the real-world data has been extracted from the Bombay Stock Exchange (BSE), an Indian premier market for financial stocks. The sample data set contains randomly selected 20 stocks, which were taken from the BSE website (www.bseindia.com). The exchange codes for the stocks are presented in Table 1.

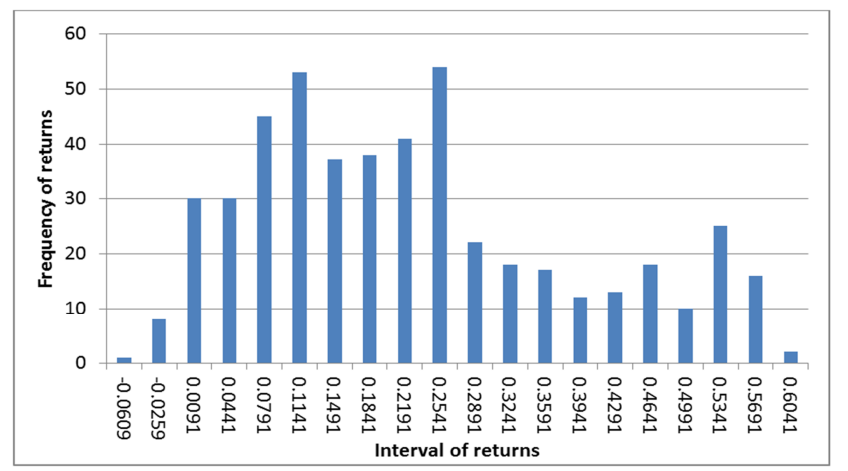

Figure 1. Frequency distribution of historical return for stock 500003.

To simulate the future returns for the stocks, the daily closing prices were used for the duration ranging from August 1, 2017 to July 25, 2019 (490 observations). Since future returns on stocks are supposed to be trapezoidal fuzzy variables, we need to compute quadruplet crisp numbers of the trapezoidal fuzzy variable for each stock. To estimate these parameters a group of expert's was formed, their technique is presented here for the first (500003) stock. Experts apprize that it can be possible to express the return 
observations graphically in the trapezoidal form (see Figure 1). The Figure 1 shows that most of the observations fall in the intervals [0.0791, 0.1141], [0.1141, 0.1491], [0.1491, $0.1841],[0.1841,0.2191]$ and $[0.2191,0.2541]$. The group of expert's advised that mid-points of the intervals [0.0791, $0.1141]$ and $[0.2191,0.2541]$ can be fixed as endpoints of the tolerance of quadruplet crisp numbers.

Table 1. The exchange codes of 20 stocks.

\begin{tabular}{|c|c|c|c|c|c|c|c|c|c|c|}
\hline Stock Code & $\begin{array}{l}S_{1} \\
500003\end{array}$ & $\begin{array}{l}S_{2} \\
500010\end{array}$ & $\begin{array}{l}S_{3} \\
500042\end{array}$ & $\begin{array}{l}S_{4} \\
500087\end{array}$ & $\begin{array}{l}S_{5} \\
500180\end{array}$ & $\begin{array}{l}S_{6} \\
500266\end{array}$ & $\begin{array}{l}S_{7} \\
500356\end{array}$ & $\begin{array}{l}S_{8} \\
500459\end{array}$ & $\begin{array}{l}S_{9} \\
500470\end{array}$ & $\begin{array}{l}S_{10} \\
500477\end{array}$ \\
\hline \multirow{2}{*}{ Stock Code } & $S_{11}$ & $S_{12} 5$ & $S_{13}$ & $S_{14}$ & $S_{15}$ & $S_{16}$ & $S_{17}$ & $S_{18}$ & $S_{19}$ & $S_{20}$ \\
\hline & 500820 & 00830 & 509480 & 522205 & 526544 & 532174 & 532977 & 533148 & 533278 & 533608 \\
\hline
\end{tabular}

Table 2. The trapezoidal fuzzy return rates of the stocks.

\begin{tabular}{lllll}
\hline Stock & Trap $(\eta)$ & Stock & Trap $(\eta)$ \\
\hline 1 & $(-0.0526,0.0966,0.2366,0.5489)$ & 11 & $(-0.0363,0.0125,0.2087,0.2912)$ \\
2 & $(-0.0656,0.0162,0.1194,0.2870)$ & 12 & $(-0.0334,0.0343,0.0908,0.2346)$ \\
3 & $(-0.1825,0.0012,0.4156,0.5220)$ & 13 & $(-0.0340,0.0683,0.2747,0.3433)$ \\
4 & $(-0.0980,0.0039,0.0874,0.1738)$ & 14 & $(-0.1294,-0.038,0.3620,0.9277)$ \\
5 & $(-0.0243,0.0453,0.1793,0.3756)$ & 15 & $(-0.1122,0.0614,0.3414,0.7617)$ \\
6 & $(-0.0083,0.1100,0.3260,0.7731)$ & 16 & $(-0.1258,-0.0075,0.1845,0.4331)$ \\
7 & $(-0.2648,-0.0180,0.1780,0.5017)$ & 17 & $(-0.1064,-0.0208,0.0973,0.177)$ \\
8 & $(0.0105,0.1652,0.2440,0.3449)$ & 18 & $(-0.1141,-0.0280,0.0720,0.3082)$ \\
9 & $(-0.1967,-0.0555,0.0615,0.3351)$ & 19 & $(-0.1368,0.0355,0.0935,0.2225)$ \\
10 & $(-0.2800,-0.1215,0.2035,0.4354)$ & 20 & $(-0.5227,0.0194,0.4354,0.5937)$ \\
\hline
\end{tabular}

Furthermore, the group of expert's advised that there can be some observations that can be affected our estimation, so by observing carefully the data, we can set values -0.0526 and 0.5489 as the minimum and maximum possible values respectively of quadruplet crisp numbers. Thus trapezoidal quadruplet crisp numbers of the first stock become [-0.0526, $0.0966,0.2366,0.5489]$. By adopting the same way trapezoidal fuzzy returns of the all 20 stocks can be estimated, which are presented in Table 2. In order to obtain the investment strategies through the proposed model, we first solve all the three single objective programming problems with all required constraints. To avoid the very small and very large proportions of the amount to be invested, we set lower and upper bound $8 \%$ and $45 \%$ respectively for the proportion as constraints in proposed modal. After solving all the three single objective programming problems with all required constraints, we get optimal objective value with respect to the constraints. The maximum portfolio return takes the value 0.28215 , the minimum risk on portfolio return takes the value 0.04156 and the maximum portfolio skewness takes the value
0.008497 with respect to the constraints. These values are taken as optimal goals in main programming problem.

\subsection{Computational Results}

In order to obtain the investment strategies through the proposed model, we present the computational results of proposed modal with optimal goals for the objectives. We set goal $g_{1}=0.28215$ for the expected portfolio return, the goal $g_{2}=0.04156$ for risk of the portfolio and the goal $g_{3}=$ 0.008497 for skewness of the portfolio. After solving the proposed optimization programming problem with respect to the all constraints, the compatible computational results of the proposed model are compressed in Table 3 . The proposed model suggested investors to invest his/her capital budget in stocks $S_{1}, S_{6}, S_{14}, S_{15}$ with the investment proportions $8 \%$, $45 \%, 39 \%, 8 \%$ respectively of his/her capital budget. In proposed investment strategies, the expected value of optimal goals will get the values $0.28215,0.04323$ and 0.00795 respectively in future. These show that we will get the expected goals almost nearly to the optimal goals.

Table 3. Computational results.

\begin{tabular}{|c|c|c|c|c|}
\hline \multirow{2}{*}{ Deviation Parameters } & $d_{1}$ & $d_{2}$ & \multicolumn{2}{|l|}{$d_{3}$} \\
\hline & $\mathbf{0}$ & 0.039 & \multicolumn{2}{|l|}{0.0005} \\
\hline \multirow{2}{*}{ Objective Parameters } & Portfolio return & Portfolio risk & \multicolumn{2}{|l|}{ Portfolio skewness } \\
\hline & 0.28215 & 0.04323 & 0.00795 & \\
\hline Optimal allocations & 0.08 & 0.45 & 0.39 & 0.08 \\
\hline
\end{tabular}

\subsection{Comparison with Existing Work}

To show the novelty of the proposed model, we compare the computational results with some existing work related to credibilistic risk measures for trapezoidal fuzzy variable. Since there are various risk measures in portfolio selection modals (such as variance, absolute deviation, entropy, value at risk etc.), so we compare outcomes of proposed model taking our risk measure with outcomes of other three models taking compatible risk measures namely semi-entropy which is presented by Zhou et al. [35], conditional value-at-risk presented by Liu et al. [21], and semi-absolute deviation 
proposed by Vercher and Bermudez [27]. We apply the same problem formulation methodology and the same solution methodology for presented model and other models using these risk measures and obtain the results. To compare the results all outcomes are presented geometrically. The comprised results of expected portfolio returns of various models are presented geometrically (see Figure 2).

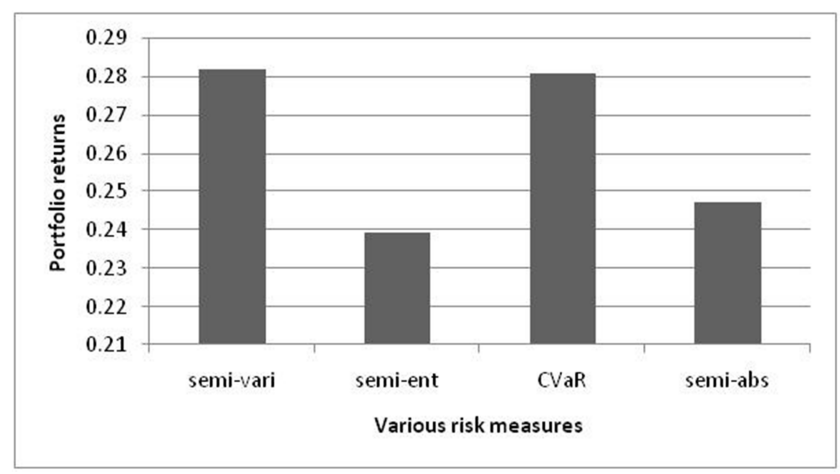

Figure 2. Comparison of expected portfolio returns using various risk measures.

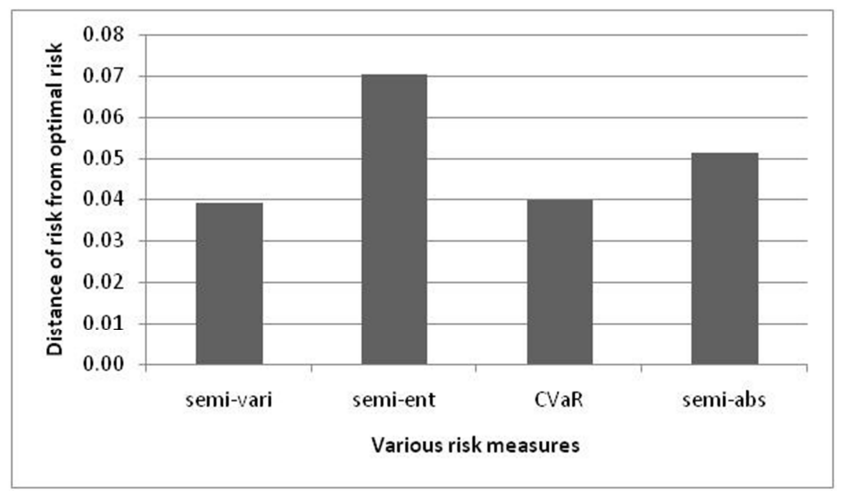

Figure 3. Comparison of risk from optimal risk using various risk measures.

We see that best expected portfolio return occurs using proposed risk measure. Similarly comprised results of risk on expected portfolio returns shows that minimum optimal risk obtains by proposed model. Also to compare various risk measure we used simple distance of future risk from optimal risk and presented this distance geometrically (see Figure 3). The Figure 3 shows that minimum risk distance finds by proposed model.

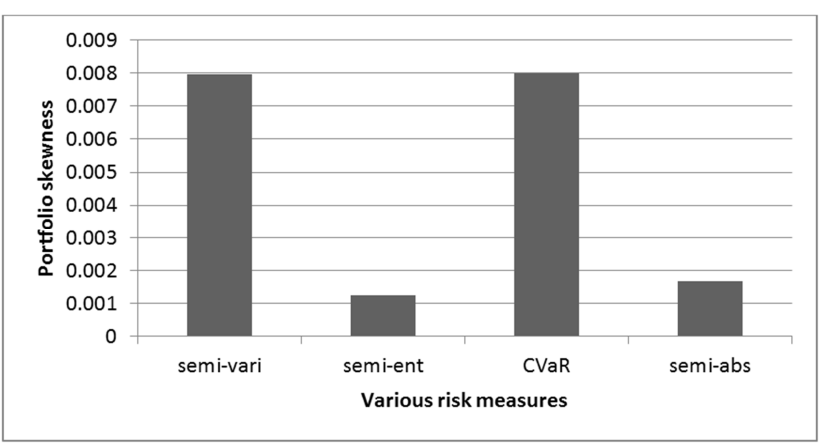

Figure 4. Comparison of expected portfolio skewness using various risk measures.
Finally we compare geometrically the portfolio skewness using various risk measure with the skewness of proposed model (see Figure 4), we find that maximum portfolio return using proposed risk measure and conditional value-at-risk measure. This numerical comparison of outcomes of various models show that presented model is best on each scale. We also compare stock proportions of various models with presented model. Comparison of optimal stocks and its allocations are presented in Table 4. Results presented in Table 4 shows that portfolio optimization model with proposed risk measure suggest investors to invest his/her capital budget in stocks $S_{1}, S_{6}, S_{14}, S_{15}$ with the investment proportions $8 \%$, $45 \%, 39 \%, 8 \%$ respectively of his/her capital budget, portfolio optimization model with semi-entropy risk measure suggest investors to invest his/her capital budget in stocks $S_{1}, S_{6}, S_{8}, S_{13}$ with the investment proportions $8 \%, 45 \%$, $39 \%, 8 \%$ respectively of his/her capital budget, portfolio optimization model with CVaR risk measure suggest investors to invest his/her capital budget in stocks $S_{1}, S_{6}, S_{14}, S_{15}$ with the investment proportions $8 \%, 39 \%, 45 \%, 8 \%$ respectively of his/her capital budget and semi-absolute deviation risk measure suggest investors to invest his/her capital budget in stocks $S_{1}, S_{6}, S_{8}, S_{15}$ with the investment proportions $8 \%$, $45 \%, 39 \%, 8 \%$ respectively of his/her capital budget. Investor can generate more portfolios with different number of stocks according to his/her satisfaction.

Table 4. Allocation of capital budget using various risk measures.

\begin{tabular}{lllll}
\hline \multirow{2}{*}{ Semi-vari } & $\mathrm{S}_{1}$ & $\mathrm{~S}_{6}$ & $\mathrm{~S}_{14}$ & $\mathrm{~S}_{15}$ \\
& 0.08 & 0.45 & 0.39 & 0.08 \\
Semi-ent & $\mathrm{S}_{1}$ & $\mathrm{~S}_{6}$ & $\mathrm{~S}_{8}$ & $\mathrm{~S}_{13}$ \\
& 0.08 & 0.45 & 0.39 & 0.08 \\
CVaR & $\mathrm{S}_{1}$ & $\mathrm{~S}_{6}$ & $\mathrm{~S}_{14}$ & $\mathrm{~S}_{15}$ \\
& 0.08 & 0.39 & 0.45 & 0.08 \\
Semi-abs & $\mathrm{S}_{1}$ & $\mathrm{~S}_{6}$ & $\mathrm{~S}_{8}$ & $\mathrm{~S}_{15}$ \\
& 0.08 & 0.45 & 0.39 & 0.08 \\
\hline
\end{tabular}

\section{Conclusions}

This paper has obtained credibilistic semi-variance of the trapezoidal fuzzy variable and applied this concept to quantify risk on future return of risky stocks and the risk on expected portfolio return. An empirical multi-criteria meansemivariance-skewness portfolio selection model has presented under a fuzzy environment. In order to obtain optimal portfolios, a multi-objective optimization has been constructed concerning mean, semivariance and skewness as objective functions with various constraints. To solve multiobjective programming problem an optimal goal programming approach has been applied. A numerical exemplification has been delivered to expound the deportment of the proposed portfolio selection model, using original historical data from the Bombay Stock Exchange, India. To show the novelty of the proposed model, we compared the computational results with some existing work related to credibilistic risk measures for trapezoidal fuzzy variable namely semi-entropy, conditional value-at-risk, and semi-absolute deviation. The computational outcomes demonstrate that the proposed portfolio optimization 
approach come out clean congenial portfolio optimization strategies, according to the investor's degree of amusement.

\section{Funding}

This research did not receive any specific grant from funding agencies in the public, commercial, or not-for-profit sectors.

\section{Acknowledgements}

We are thankful to respective referees for their pointed explanation of this paper and serious comments, indications and suggestions to improve our work.

\section{References}

[1] H. Markowitz, Portfolio selection, J. Finance 7 (1952) 77-91.

[2] H. Markowitz, Portfolio Selection: Efficient Diversification of Investments, Wiley, New York, 1959.

[3] F. Choobineh, D. Branting, A simple approximation for semivariance, European J. Oper. Res. 27 (1986) 364-370.

[4] H. Markowitz, Computation of mean-semivariance efficient sets by the critical line algorithm, Ann. Oper. Res. 45 (1993) 307-317.

[5] P. D. Kaplan, R. H. Alldredge, Semivariance in risk-based index construction: quantidex global indexes, The J. Investing 6 (1997) 82-87.

[6] H. Tanaka, P. Guo, Portfolio selection based on upper and lower exponential possibility distributions, European J. Oper. Res. 114 (1999) 115-126.

[7] H. Tanaka, P. Guo, I. B. Türksen, Portfolio selection based on fuzzy probabilities and possibility distributions, Fuzzy Sets and Systems 111 (2000) 387-397.

[8] C. Carlsson, R. Fullér, P. Majlender, A possibilistic approach to selecting portfolios with highest utility score, Fuzzy sets and systems, 131 (1) (2002), 13-21.

[9] Huang, X., 2007. Portfolio selection with fuzzy returns. Journal of Intelligent \& Fuzzy Systems, 18 (4), pp. 383-390.

[10] Liu, B. and Liu, Y. K., 2002. Expected value of fuzzy variable and fuzzy expected value models. IEEE transactions on Fuzzy Systems, 10 (4), pp. 445-450.

[11] Huang, X., 2008. Mean-semivariance models for fuzzy portfolio selection. Journal of computational and applied mathematics, 217 (1), pp. 1-8.

[12] Qin Z, Li X, Ji X (2009) Portfolio selection based on fuzzy cross-entropy. J Comput Appl Math 228 (1): 139-149.

[13] Qin Z, Wang ZD, Li X (2013) Mean-semivariance models for portfolio optimization with mixed uncertainty of fuzziness and randomness. Int J Uncertain Fuzziness Knowl-Based Syst 21 (1): $127-139$.

[14] Jalota Hemant, Manoj Thakur, and Garima Mittal. "Modelling and constructing membership function for uncertain portfolio parameters: A credibilistic framework." Expert Systems with Applications 71 (2017): 40-56.
[15] Liu Yong-Jun, and Wei-Guo Zhang. "Fuzzy portfolio selection model with real features and different decision behaviors." Fuzzy Optimization and Decision Making 17, no. 3 (2018): 317-336.

[16] Zhang, Peng. "Multi-period possibilistic mean semivariance portfolio selection with cardinality constraints and its algorithm." Journal of Mathematical Modelling and Algorithms in Operations Research 14, no. 2 (2015): 239-253.

[17] Liu, Yongjun, Wei-Guo Zhang, and Pankaj Gupta. "Multiperiod Portfolio Performance Evaluation Model Based on Possibility Theory." IEEE Transactions on Fuzzy Systems (2019).

[18] Zhang, Peng, and Bi-Yu Peng. "Credibilitic Multiperiod Mean Semivariance Portfolio Selection with Transaction Costs." INDUSTRIAL ENGINEERING AND MANAGEMENT SYSTEMS 17, no. 3 (2018): 464-478.

[19] M. K. Mehlawat, Credibilistic mean-entropy models for multiperiod portfolio selection with multi-choice aspiration levels, Information Sciences, 345 (2016), 9-26.

[20] E. Vercher, J. D. Bermúdez, Measuring uncertainty in the portfolio selection problem. In The Mathematics of the Uncertain, Cham (2018), 765-775.

[21] N. Liu, Y. Chen, Y. Liu, Optimizing portfolio selection problems under credibilistic CVaR criterion, Journal of Intelligent \& Fuzzy Systems, 34 (1) (2018), 335-347.

[22] Gupta Pankaj, Mukesh Kumar Mehlawat, Arun Kumar, Sanjay Yadav, and Abha Aggarwal. "A Credibilistic Fuzzy DEA Approach for Portfolio Efficiency Evaluation and Rebalancing Toward Benchmark Portfolios Using Positive and Negative Returns." International Journal of Fuzzy Systems 22, no. 3 (2020): 824-843.

[23] Mehlawat, Mukesh Kumar, Pankaj Gupta, Arun Kumar, Sanjay Yadav, and Abha Aggarwal. "Multi-Objective Fuzzy Portfolio Performance Evaluation Using Data Envelopment Analysis Under Credibilistic Framework." IEEE Transactions on Fuzzy Systems (2020).

[24] X. Li, Z. Qin, S. Kar, Mean-variance-skewness model for portfolio selection with fuzzy returns, European Journal of Operational Research, 202 (1) (2010), 239-247.

[25] J. S. Kamdem, C. T. Deffo, L. A. Fono, Moments and semimoments for fuzzy portfolio selection, Insurance: Mathematics and Economics, 51 (3) (2012), 517-530.

[26] S. Barak, M. Abessi, M. Modarres, Fuzzy turnover rate chance constraints portfolio model, European Journal of Operational Research, 228 (1) (2013), 141-147.

[27] E. Vercher, J. D. Bermúdez, Portfolio optimization using a credibility mean-absolute semi-deviation model, Expert Systems with Applications, 42 (20) (2015), 7121-7131.

[28] Z. Qin, Credibilistic mean-variance-skewness model, In Uncertain Portfolio Optimization, Singapore (2016), 29-52.

[29] A. Ray, S. K. Majumder, Multi objective mean-varianceskewness model with Burg's entropy and fuzzy return for portfolio optimization, Opsearch, 55 (1) (2018), 107-133.

[30] M. Rahimi, P. Kumar, Portfolio optimization based on fuzzy entropy, International Journal on Interactive Design and Manufacturing (IJIDeM), 13 (2) (2019), 531-536. 
[31] Zhang, W. G. and Liu, Y. J.: Credibilitic mean-variance model for multi-period portfolio selection problem with risk control. OR Spectr. 36 (1), 113-132 (2014)

[32] Qin, Zhongfeng, Meilin Wen, and Changchao Gu. "Meanabsolute deviation portfolio selection model with fuzzy returns." Iranian Journal of Fuzzy Systems 8, no. 4 (2011): 6175 .

[33] Deng, X., Zhao, J. and Li, Z.: Sensitivity Analysis of the Fuzzy Mean-Entropy Portfolio Model with Transaction Costs Based on Credibility Theory. Int. J. Fuzzy Syst. 20 (1), 209218 (2018).

[34] B. Wang, S. Wang, J. Watada, Fuzzy-portfolio-selection models with value-at-risk, IEEE Transactions on Fuzzy Systems, 19 (4) (2011), 758-769.

[35] Zhou, Jiandong, Xiang Li, and Witold Pedrycz. "Mean-semi- entropy models of fuzzy portfolio selection." IEEE Transactions on Fuzzy Systems 24, no. 6 (2016): 1627-1636.

[36] LA. Zadeh, Fuzzy sets as a basis for a theory of possibility, Fuzzy Sets Syst 1 (1978), 3-28.

[37] X. Li, BD Liu, A sufficient and necessary condition for credibility measures, Int J Uncertain Fuzziness Knowl-Based Syst 14 (5) (2006), 527-535.

[38] BD. Liu, Uncertainty theory, Springer, Berlin, 2nd edn. (2007).

[39] BD. Liu, Theory and practice of uncertain programming, Physica-Verlag, Heidelberg, (2002).

[40] Y. K., Liu, B. Liu, Expected value operator of random fuzzy variable and random fuzzy expected value models, International Journal of Uncertainty, Fuzziness and Knowledge-Based Systems, 11 (2) (2003), 195-215. 